\title{
Correction to: Efficacy, tolerability and safety of darbepoetin alfa injection for the treatment of anemia associated with chronic kidney disease (CKD) undergoing dialysis: a randomized, phase-III trial
}

Shubhadeep D. Sinha ${ }^{1}$, Vamsi Krishna Bandi ${ }^{1}$, Bala Reddy Bheemareddy ${ }^{1}$, Pankaj Thakur ${ }^{1 *}$, Sreenivasa Chary' Kalpana Mehta ${ }^{2}$, Vikranth Reddy Pinnamareddy ${ }^{3}$, Rajendra Pandey ${ }^{4}$, Subhramanyam Sreepada ${ }^{5}$ and Santosh Durugkar ${ }^{6}$

Correction to: BMC Nephrol (2019) 20:90

https://doi.org/10.1186/s12882-019-1209-1

Following publication of the original article [1], the authors reported errors in the presentation of Tables 2, 4 and 5. Additionally, the authors reported an error in the last paragraph of the 'Safety assessment' section and an error in the first paragraph of the 'Discussion' section. In this Correction the incorrect and correct version of $\mathrm{Ta}$ bles 2, 4 and 5 and the incorrect and correct version of the sentences in the 'Safety assessment' and 'Discussion' section are shown.

Originally Table 2 was published as:

Table 2 Mean Hb levels ( $\mathrm{g} / \mathrm{dL})$ and mean change in hemoglobin from Baseline to EOC - Dialysis, ITT Population $(N=126)$

\begin{tabular}{|c|c|c|c|c|}
\hline \multirow[t]{2}{*}{ Statistics } & \multicolumn{2}{|l|}{ ITT Population $(N=126)$} & \multicolumn{2}{|l|}{ PP Population $(N=93)$} \\
\hline & Darbepoetin alfa $(n=63)$ & Erythropoietin alfa $(n=63)$ & Darbepoetin alfa $(n=47)$ & Erythropoietin alfa $(n=46)$ \\
\hline \multicolumn{5}{|l|}{ Baseline } \\
\hline$n$ & 56 & 53 & 47 & 46 \\
\hline Mean (SD) & $8.39(0.90)$ & $8.80(0.89)$ & $8.39(0.85)$ & $8.72(0.91)$ \\
\hline \multicolumn{5}{|c|}{ End of first evaluation visit } \\
\hline$n$ & 55 & 51 & 47 & 46 \\
\hline Mean (SD) & $10.20(1.74)$ & $10.61(1.55)$ & $10.33(1.42)$ & $10.90(0.95)$ \\
\hline \multicolumn{5}{|c|}{ Within group comparison } \\
\hline$p$-value\# & $<.0001$ & $<.0001$ & $<.0001$ & $<.0001$ \\
\hline Mean change & 1.84 & 1.85 & 1.94 & 2.18 \\
\hline $95 \% \mathrm{Cl}$ & {$[1.36-2.32]$} & {$[1.37-2.33]$} & {$[1.48-2.40]$} & {$[1.84-2.53]$} \\
\hline \multicolumn{5}{|c|}{ Between group comparison } \\
\hline Mean change & -0.01 & & -0.24 & \\
\hline $95 \% \mathrm{Cl}$ & {$[-0.68-0.66]$} & & {$[-0.81-0.32]$} & \\
\hline
\end{tabular}

\footnotetext{
* Correspondence: Pankaj.Thakur@heterodrugs.com

${ }^{1}$ Clinical Development and Medical Affairs, Hetero Group, Hetero Corporate,

7-2-A2, Industrial Estates, Sanath Nagar, Hyderabad, Andhra Pradesh, India

Full list of author information is available at the end of the article
}

(c) The Author(s). 2019 Open Access This article is distributed under the terms of the Creative Commons Attribution 4.0 International License (http://creativecommons.org/licenses/by/4.0/), which permits unrestricted use, distribution, and reproduction in any medium, provided you give appropriate credit to the original author(s) and the source, provide a link to the Creative Commons license, and indicate if changes were made. The Creative Commons Public Domain Dedication waiver (http://creativecommons.org/publicdomain/zero/1.0/) applies to the data made available in this article, unless otherwise stated. 
Table 2 Mean Hb levels ( $\mathrm{g} / \mathrm{dL})$ and mean change in hemoglobin from Baseline to EOC - Dialysis, ITT Population $(N=126)$ (Continued)

\begin{tabular}{llll}
\hline Statistics & ITT Population $(N=126)$ & PP Population $(N=93)$ & $\frac{\text { Darbepoetin alfa }(n=47)}{n n y}$ \\
\cline { 2 - 4 } & Darbepoetin alfa $(n=63)$ & Erythropoietin alfa $(n=63)$ & 0.3985 \\
\hline
\end{tabular}

$N$ number of subject at each visit, $N$ total number of subjects, $I T T$ Intent to treat, $P P$ Per protocol

${ }_{* *} p$-values were obtained using Paired t Test for mean (two tailed, a 0.05)

${ }^{* *} p$-values were obtained using Unpaired t Test for mean change (two tailed, $a=0.05$ )

Note: Patients taken where $\mathrm{Hb}<10$ at Screening

The correct version of Table 2, with the corrected sections indicated in bold:

Table 2 Mean Hb levels $(\mathrm{g} / \mathrm{dL})$ and mean change in hemoglobin from Baseline to EOC - Dialysis, ITT Population $(N=126)$

\begin{tabular}{|c|c|c|c|c|}
\hline \multirow[t]{2}{*}{ Statistics } & \multicolumn{2}{|l|}{ ITT Population $(N=126)$} & \multicolumn{2}{|l|}{ PP Population $(N=93)$} \\
\hline & Darbepoetin alfa $(n=63)$ & Erythropoietin alfa $(n=63)$ & Darbepoetin alfa $(n=47)$ & Erythropoietin alfa $(n=46)$ \\
\hline \multicolumn{5}{|l|}{ Baseline } \\
\hline$n$ & 56 & 53 & 47 & 46 \\
\hline Mean (SD) & $8.39(0.90)$ & $8.80(0.89)$ & $8.39(0.85)$ & $8.72(0.91)$ \\
\hline \multicolumn{5}{|c|}{ End of first evaluation visit } \\
\hline$n$ & 55 & 51 & 47 & 46 \\
\hline Mean (SD) & $10.20(1.74)$ & $10.61(1.55)$ & $10.33(1.42)$ & $10.90(0.95)$ \\
\hline \multicolumn{5}{|c|}{ Within group comparison } \\
\hline$p$-value & $<.0001$ & $<.0001$ & $<.0001$ & $<.0001$ \\
\hline Mean change & 1.84 & 1.85 & 1.94 & 2.18 \\
\hline $95 \% \mathrm{Cl}$ & {$[1.36-2.32]$} & {$[1.37-2.33]$} & {$[1.48-2.40]$} & {$[1.84-2.53]$} \\
\hline \multicolumn{5}{|c|}{ Between group comparison } \\
\hline Mean change & -0.01 & & -0.24 & \\
\hline $95 \% \mathrm{Cl}$ & {$[-0.68-0.66]$} & & {$[-0.81-0.32]$} & \\
\hline$p$-value ${ }^{* *}$ & 0.9703 & & 0.3985 & \\
\hline
\end{tabular}

$\boldsymbol{n}$ number of subject at each visit, $N$ total number of subjects, $I T T$ Intent to treat, $P P$ Per protocol, $\boldsymbol{H} \boldsymbol{b}$ Hemoglobin

\# $p$-values were obtained using Paired $t$ Test for mean (two tailed, $\mathbf{a}=\mathbf{0 . 0 5}$ )

${ }^{* *} p$-values were obtained using Unpaired t Test for mean change (two tailed, $a=0.05$ )

Note: Patients taken where $\mathrm{Hb}<10$ at Screening

\section{Originally Table 4 was published as:}

Table 4 Mean change in hemoglobin levels $(\mathrm{g} / \mathrm{dL})$ from baseline to week-4

\begin{tabular}{|c|c|c|c|c|}
\hline \multirow[t]{2}{*}{ Statistics } & \multicolumn{2}{|l|}{ ITT Population $(N=126)$} & \multicolumn{2}{|l|}{ PP Population $(N=93)$} \\
\hline & Darbepoetin alfa $(n=63)$ & Erythropoietin alfa $(n=63)$ & Darbepoetin alfa $(n=47)$ & Erythropoietin alfa $(n=46)$ \\
\hline \multicolumn{5}{|l|}{ Baseline } \\
\hline$n$ & 56 & 53 & 47 & 46 \\
\hline Mean (SD) & $8.39(0.90)$ & $8.80(0.89)$ & $8.39(0.85)$ & $8.72(0.91)$ \\
\hline \multicolumn{5}{|l|}{ Week-4 } \\
\hline$n$ & 55 & 50 & 47 & 45 \\
\hline Mean (SD) & $8.66(1.24)$ & $9.50(1.81)$ & $8.68(1.13)$ & $9.62(1.71)$ \\
\hline \multicolumn{5}{|c|}{ Within group comparison } \\
\hline$p$-value ${ }^{*}$ & 0.0566 & 0.0019 & 0.0473 & 0.0002 \\
\hline Mean change & 0.30 & 0.74 & 0.29 & 0.91 \\
\hline $95 \% \mathrm{Cl}$ & {$[-0.01-0.61]$} & {$[0.29-1.19]$} & {$[0.00-0.57]$} & {$[0.45-1.36]$} \\
\hline \multicolumn{5}{|c|}{ Between group comparison } \\
\hline Mean change & -0.44 & & -0.62 & \\
\hline
\end{tabular}


Table 4 Mean change in hemoglobin levels ( $\mathrm{g} / \mathrm{dL}$ ) from baseline to week-4 (Continued)

\begin{tabular}{|c|c|c|c|c|}
\hline \multirow[t]{2}{*}{ Statistics } & \multicolumn{2}{|l|}{ ITT Population $(N=126)$} & \multicolumn{2}{|l|}{ PP Population $(N=93)$} \\
\hline & Darbepoetin alfa $(n=63)$ & Erythropoietin alfa $(n=63)$ & Darbepoetin alfa $(n=47)$ & Erythropoietin alfa $(n=46)$ \\
\hline $95 \% \mathrm{Cl}$ & {$[-0.97-0.09]$} & & {$[-1.14-0.10]$} & \\
\hline$p$-value ${ }^{* *}$ & 0.1057 & & 0.0209 & \\
\hline
\end{tabular}

$n$ number of subject at each visit; $N$ total number of subjects, $I T T$ Intent to treat, $P P$ Per protocol

${ }^{*} p$-value were obtained using Paired t Test for mean (two tailed, $a=0.05$ )

${ }^{* *} p$-value were obtained using Unpaired $t$ Test for mean change (two tailed, $a=0.05$ )

Note: Patients taken where $\mathrm{Hb}<10$ at Screening

The correct version of Table 4, with the corrected sections indicated in bold:

Table 4 Mean change in hemoglobin levels $(\mathrm{g} / \mathrm{dL})$ from baseline to week-4

\begin{tabular}{|c|c|c|c|c|}
\hline \multirow[t]{2}{*}{ Statistics } & \multicolumn{2}{|l|}{ ITT Population $(N=126)$} & \multicolumn{2}{|l|}{ PP Population $(N=93)$} \\
\hline & Darbepoetin alfa $(n=63)$ & Erythropoietin alfa $(n=63)$ & Darbepoetin alfa $(n=47)$ & Erythropoietin alfa $(n=46)$ \\
\hline \multicolumn{5}{|l|}{ Baseline } \\
\hline$n$ & 56 & 53 & 47 & 46 \\
\hline Mean (SD) & $8.39(0.90)$ & $8.80(0.89)$ & $8.39(0.85)$ & $8.72(0.91)$ \\
\hline \multicolumn{5}{|l|}{ Week-4 } \\
\hline$n$ & 55 & 50 & 47 & 45 \\
\hline Mean (SD) & $8.66(1.24)$ & $9.50(1.81)$ & $8.68(1.13)$ & $9.62(1.71)$ \\
\hline \multicolumn{5}{|c|}{ Within group comparison } \\
\hline$p$-value ${ }^{*}$ & 0.0566 & 0.0019 & 0.0473 & 0.0002 \\
\hline Mean change & 0.30 & 0.74 & 0.29 & 0.91 \\
\hline $95 \% \mathrm{Cl}$ & {$[-0.01-0.61]$} & {$[0.29-1.19]$} & {$[0.00-0.57]$} & {$[0.45-1.36]$} \\
\hline \multicolumn{5}{|c|}{ Between group comparison } \\
\hline Mean change & -0.44 & & -0.62 & \\
\hline $95 \% \mathrm{Cl}$ & {$[-0.97-0.09]$} & & {$[-1.14-0.10]$} & \\
\hline$p$-value ${ }^{* *}$ & 0.1057 & & 0.0209 & \\
\hline
\end{tabular}

$n$ number of subject at each visit; $N$ total number of subjects, ITT Intent to treat, $P P$ Per protocol, $\mathbf{H b}$ Hemoglobin

${ }^{*} p$-value were obtained using Paired t Test for mean (two tailed, $\mathbf{a}=\mathbf{0 . 0 5}$ )

${ }^{* *} p$-value were obtained using Unpaired $t$ Test for mean change (two tailed, $\mathbf{a}=\mathbf{0 . 0 5}$ )

Note: Patients taken where $\mathrm{Hb}<10$ at Screening

\section{Originally Table 5 was published as:}

Table 5 Time to initially attained target $\mathrm{Hb}$ level $(10-12 \mathrm{~g} / \mathrm{dL})$ and proportion of patients attained target $\mathrm{Hb}$ level (10-12 g/dL) at EOC and EOM

\begin{tabular}{|c|c|c|c|c|}
\hline \multirow[t]{2}{*}{ Parameter } & \multicolumn{2}{|l|}{ ITT Population $(N=126)$} & \multicolumn{2}{|l|}{ PP Population $(N=93)$} \\
\hline & Darbepoetin alfa $(n=63)$ & Erythropoietin alfa $(n=63)$ & Darbepoetin alfa $(n=47)$ & Erythropoietin alfa $(n=46$ \\
\hline \multicolumn{5}{|c|}{ Number of weeks to initially attain target $\mathrm{Hb}$} \\
\hline Median $(95 \% \mathrm{Cl})$ & $9.00(7.00-11.00)$ & $7.00(4.00-9.00)$ & $9.00(7.00-10.00)$ & $7.00(4.00-8.00)$ \\
\hline \multicolumn{5}{|c|}{ No. of Patients initially attained target $\mathrm{Hb}$ level } \\
\hline N (\%) & $44(78.57)$ & $43(82.69)$ & $40(85.10)$ & $41(89.13)$ \\
\hline Hazard Ratio $(95 \% \mathrm{Cl})$ & $0.807(0.53-1.23)$ & & $0.778(0.50-1.21)$ & \\
\hline$P$ Value & 0.3212 & & 0.2608 & \\
\hline
\end{tabular}

No. of patients attained target $\mathrm{Hb}$ level at EOC

\begin{tabular}{|c|c|c|c|}
\hline$N(\%)$ & $33(52.38)$ & $32(68.08)$ & $32(69.56)$ \\
\hline Odd ratios $(95 \% \mathrm{Cl})$ & $0.9559(0.46-1.99)$ & $0.9410(0.39-2.30)$ & \\
\hline$P$ value & 0.9038 & 0.8938 & \\
\hline
\end{tabular}


Table 5 Time to initially attained target $\mathrm{Hb}$ level $(10-12 \mathrm{~g} / \mathrm{dL})$ and proportion of patients attained target $\mathrm{Hb}$ level (10-12 $\mathrm{g} / \mathrm{dL})$ at EOC and EOM (Continued)

\begin{tabular}{|c|c|c|c|c|}
\hline \multirow[t]{2}{*}{ Parameter } & \multicolumn{2}{|l|}{ ITT Population $(N=126)$} & \multicolumn{2}{|l|}{ PP Population $(N=93)$} \\
\hline & Darbepoetin alfa $(n=63)$ & Erythropoietin alfa $(n=63)$ & Darbepoetin alfa $(n=47)$ & Erythropoietin alfa $(n=46)$ \\
\hline \multicolumn{5}{|c|}{ No. of patients maintained target $\mathrm{Hb}$ level at EOM } \\
\hline$(\%)$ & $24(38.10)$ & $36(57.14)$ & $15(34.09)$ & $23(57.50)$ \\
\hline Odd ratios $(95 \% \mathrm{Cl})$ & $0.5748(0.26-1.25)$ & & $0.4567(0.17-1.22)$ & \\
\hline$P$ Value & 0.1621 & & 0.1180 & \\
\hline
\end{tabular}

EOC End of correction, EOM End of maintenance, ITT Intent to treat, $P P$ Per protocol, $H b$ Hemoglobin

The correct version of Table 5, with the corrected sections indicated in bold:

Table 5 Time to initially attained target $\mathrm{Hb}$ level $(10-12 \mathrm{~g} / \mathrm{dL})$ and proportion of patients attained target $\mathrm{Hb}$ level (10-12 g/dL) at EOC and EOM

\begin{tabular}{|c|c|c|c|c|}
\hline \multirow[t]{2}{*}{ Parameter } & \multicolumn{2}{|l|}{ ITT Population $(N=126)$} & \multicolumn{2}{|l|}{ PP Population $(N=93)$} \\
\hline & Darbepoetin alfa $(n=63)$ & Erythropoietin alfa $(n=63)$ & Darbepoetin alfa $(n=47)$ & Erythropoietin alfa $(n=46)$ \\
\hline \multicolumn{5}{|c|}{ Number of weeks to initially attain target $\mathrm{Hb}$} \\
\hline Median $(95 \% \mathrm{Cl})$ & $9.00(7.00-11.00)$ & $7.00(4.00-9.00)$ & $9.00(7.00-10.00)$ & $7.00(4.00-8.00)$ \\
\hline \multicolumn{5}{|c|}{ No. of Patients initially attained target $\mathrm{Hb}$ level } \\
\hline N (\%) & $44(78.57)$ & $43(82.69)$ & $40(85.10)$ & $41(89.13)$ \\
\hline Hazard Ratio $(95 \% \mathrm{Cl})$ & $0.807(0.53-1.23)$ & & $0.778(0.50-1.21)$ & \\
\hline$p$-value & 0.3212 & & 0.2608 & \\
\hline \multicolumn{5}{|c|}{ No. of patients attained target $\mathrm{Hb}$ level at EOC } \\
\hline N (\%) & $33(52.38)$ & $31(49.2)$ & $32(68.08)$ & $32(69.56)$ \\
\hline Odd ratios $(95 \% \mathrm{Cl})$ & $0.9559(0.46-1.99)$ & & $0.9410(0.39-2.30)$ & \\
\hline$p$-value & 0.9038 & & 0.8938 & \\
\hline \multicolumn{5}{|c|}{ No. of patients maintained target $\mathrm{Hb}$ level at EOM } \\
\hline$(\%)$ & $24(38.10)$ & $36(57.14)$ & $15(34.09)$ & $23(57.50)$ \\
\hline Odd ratios $(95 \% \mathrm{Cl})$ & $0.5748(0.26-1.25)$ & & $0.4567(0.17-1.22)$ & \\
\hline$p$-value & 0.1621 & & 0.1180 & \\
\hline
\end{tabular}

EOC End of correction, EOM End of maintenance, ITT Intent to treat, PP Per protocol, $\mathrm{Hb}$ Hemoglobin

Originally the last paragraph of the 'Safety assessment' section was published as:

- Altogether, DA- $\alpha$ had a similar safety profile to that of EPO and no antibody formation was identified.

The correct presentation of the last paragraph of the 'Safety assessment' section, with the corrected words indicated in bold:

- Altogether, DA- $\alpha$ had a similar safety profile to that of EPO and no anti-drug antibody formation was identified.

Originally two sentences in the first paragraph of the 'Discussion' section were published as:
- Evaluating the iron availability for erythropoeisis is crucial in treating anaemia patients with CKD.Iron deficiency can interfere with the response to EPO and DA- $\alpha$ and affecting the efficacy

The correct presentation of two sentences in the first paragraph of the 'Discussion' section, with the corrected words indicated in bold:

- Evaluating the iron availability for erythropoeisis is crucial in treating anaemia patients with CKD. Iron deficiency can interfere with the response to EPO and DA- $\alpha$ and affecting the efficacy

\section{Author details}

${ }^{1}$ Clinical Development and Medical Affairs, Hetero Group, Hetero Corporate, 7-2-A2, Industrial Estates, Sanath Nagar, Hyderabad, Andhra Pradesh, India. 
${ }^{2}$ Department of Nephrology, B.L.Y Nair Hospital, A.L Nair Road, Mumbai, Maharashtra, India. ${ }^{3}$ Care Hospitals, Road No. 1, Banjara Hills, Hyderabad, Andhra Pradesh, India. ${ }^{4}$ Department of Nephrology, Institute of Post Graduate Medical Education and Research Kolkata, 244 A.J.C Bose Road, Kolkata, West Bengal, India. ${ }^{5}$ Sri Raghavendra Hospital, 1-7-100, Opp. Round Building, Kamala Nagar, ECIL Cross Road, ECIL, Hyderabad, Andhra Pradesh 500062, India. ${ }^{6}$ Ashwini Hospital and Ramakanth Heart Care Center, Shivaji Nagar, Nanded, Maharashtra, India.

\section{Published online: 19 November 2019}

\section{Reference}

1. Sinha, et al. Efficacy, tolerability and safety of darbepoetin alfa injection for the treatment of anemia associated with chronic kidney disease (CKD) undergoing dialysis: a randomized, phase-III trial. BMC Nephrol. 2019;20:90, https://doi.org/10.1186/s12882-019-1209-1. 